\title{
CORRIGENDUM
}

\section{Successful overweight prevention in adolescents by increasing physical activity: a 4-year randomized controlled intervention}

C Simon, B Schweitzer, M Oujaa, A Wagner, D Arveiler, E Triby, N Copin, S Blanc and C Platat International Journal of Obesity (2008) 32, 1606; doi:10.1038/ijo.2008.138

Correction to: Int J Obes (2008) 32, 1489-1498;

doi:10.1038/ijo.2008.99; published online 15 July 2008

Since the publication of the above paper, the authors have noticed an omission in the legend of Figure 2 and they

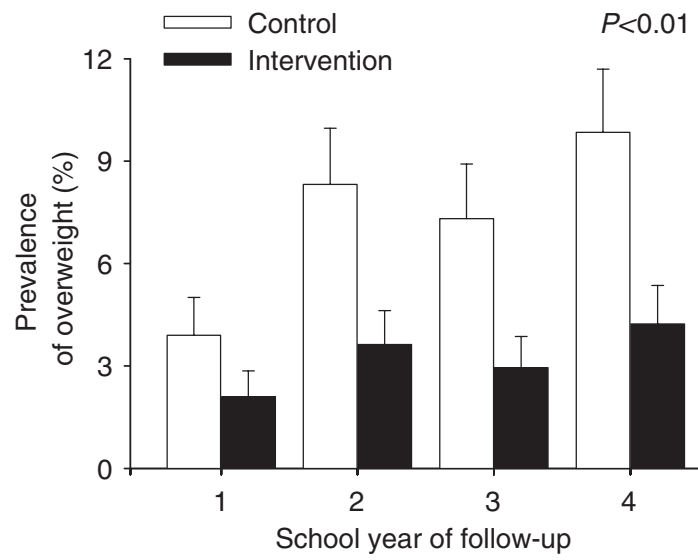

Figure 2 Prevalence of overweight in initially non-overweight participants, taking into account the cluster randomization design, adjusted for gender, sexual maturity, socioeconomic status, baseline participation to sports club, baseline stratification and their interactions with time. Overweight was defined according to the International Obesity Task Force. ${ }^{32}$ The number of initially non-overweight students was 731 at baseline, 653 at 2 years, 605 at 3 years and 571 at 4 years. $P$-value is given for intervention effect. wish to modify it slightly. The amended Figure 2 is reproduced below.

The authors apologize for the errors. 